\title{
ESFERAS PÚBLICAS EM CONFLITO: CONTRIBUIÇÕES DE NANCY FRASER AO CONCEITO DE ESFERA PÚBLICA
}

\author{
LAISA MARRA ${ }^{1}$
}

RESUMO: O artigo objetiva apresentar uma síntese das contribuições da pesquisadora feminista Nancy Fraser ao conceito de esfera pública, tendo como eixo da discussão as críticas feitas pela autora a quatro pressupostos habermasianos de tal conceito. Para tanto, apresentam-se brevemente as formulações de Jürgen Habermas, para depois problematizá-las conforme Fraser. Ademais, concordando-se com Michael Warner, a defesa que faz Fraser da ideia de "contra-públicos subalternos" é questionada por simplificar a noção de subalternidade. Por fim, avalia-se a utilidade das questões levantadas por Fraser para se pensar os problemas de desigualdades e liberalismo enfrentados pelas democracias existentes.

PALAVRAS-CHAVE: Esfera pública. Nancy Fraser. Jürgen Habermas. Crítica feminista.

ABSTRACT: The paper aims at presenting a synthesis of contributions made by feminist researcher Nancy Fraser to the concept of public sphere, having as its axis of discussion the problems pointed out by the author to four Habermasian presuppositions of such notion. To that end, the formulations of Jürgen Habermas are briefly presented, and later problematized according to Fraser. In addition, and according to Michael Warner, Fraser's defense of the idea of "subaltern counterpublics" is questioned for simplifying the notion of subalternity. Finally, it is evaluated the usefulness of the questions raised by Fraser in order to consider the problems of inequalities and liberalism faced by existing democracies.

KEYWORDS: Public sphere. Nancy Fraser. Jürgen Habermas. Feminist criticism.

Logo no início do prefácio à edição de 1990 de Mudança Estrutural na Esfera Pública, Jürgen Habermas (2014) reconhece que seu livro se tornou, nas décadas que se seguiram à primeira publicação, em 1962, uma espécie de manual incontornável pela crítica dedicada a compreender o conceito de esfera pública. Portanto, pensar os problemas levantados por Nancy Fraser (1990) sobre esse conceito nas democracias contemporâneas, objetivo principal deste artigo, significa, inevitavelmente, situar a autora em seu diálogo com Habermas (2014); afinal, o artigo de Fraser (1990), "Rethinking the public sphere", está diretamente ligado ao congresso Habermas and the Public Sphere, ocorrido em 1989 na Carolina do Norte (EUA).

É importante deixar claro, desde já, que Habermas (2014) e Fraser (1990) têm abordagens metodológicas distintas, como veremos adiante. De qualquer modo, vale enfatizar

\footnotetext{
${ }^{1}$ Doutoranda em Teoria da Literatura e Literatura Comparada pelo Programa de Pós-graduação em Estudos Literários da Universidade Federal de Minas Gerais (UFMG). E-mail: laisamarra@gmail.com.
} 
que a ambição desta breve revisão bibliográfica ${ }^{2}$ está em, principalmente, compreender como a crítica de Nancy Fraser (1990) à concepção habermasiana de esfera pública contribui para a problematização do conceito nas democracias contemporâneas. Para tanto, iniciarei este texto com um esboço da ideia habermasiana de esfera pública para depois passarmos aos questionamentos de Fraser (1990).

Habermas (2014) procura, em seu trabalho, imaginar a complexidade de uma sociedade em transição, da era absolutista para a Modernidade, e seus efeitos na construção da democracia moderna. Como se sabe, esse lento processo de configuração e estabelecimento da modernidade ocidental não envolveu apenas a transformação dos modos de produção e formas de governo, mas também a ascensão de novas formas de sociabilidade, bem como de novas categorias de pensamento, como o individualismo. Centrado na emergência da Modernidade na Europa ocidental (mais especificamente Inglaterra, França e Alemanha), Habermas teoriza sobre a interrelação entre o Estado e, em sentido amplo, a sociedade civil (incluindo o mercado privatizado), a qual poderia se manifestar criticamente através da esfera pública. Nas palavras de Habermas (2014, p. 135):

A esfera pública burguesa pode ser entendida, antes de mais nada, como a esfera de pessoas privadas que se reúnem em um público. Elas reivindicam imediatamente a esfera pública, regulamentada pela autoridade, contra o próprio poder público, de modo a debater com ela as regras universais das relações vigentes na esfera da circulação de mercadorias e do trabalho social - essencialmente privatizada, mas publicamente relevante.

No trecho citado, o autor sintetiza a noção de esfera pública em sua dependência com a delimitação contrastante entre o público e o privado. Por pessoas privadas, entendem-se grupos de pessoas inseridos na lógica do individualismo ocidental, categoria histórico-filosófica observável em sociedades cristãs pré-capitalistas, mas apenas estabilizada com a consolidação da burguesia (DUMONT, 1985). Assim, essas pessoas privadas são, a priori, indivíduos modernos, vivendo em compasso com a urbanização dos espaços - o que pressupõe a multiplicação de lugares de encontro, tais como salões e cafés, comuns na Inglaterra e na França do século XVIII (HABERMAS, 2014).

Além disso, Habermas (2014) trata do papel essencial da imprensa para a formação do público, no geral, e para a circulação regular de notícias e ideias, motivando discussões

\footnotetext{
${ }^{2}$ Logo, não se ambiciona levantar o "estado do conhecimento" sobre a esfera pública, uma vez que isso implicaria em um trabalho mais extenso e aprofundado de pesquisa bibliográfica, o que é impossível, dado o limite deste texto e a enorme quantidade de discussões importantes em torno do tema nas últimas décadas.
} 
racionais entre os leitores informados, entre outras coisas, sobre a atuação do Estado no que passa a ser considerado domínio privado. A ênfase dada por Habermas (2014) na deliberação racional entre indivíduos, atuando de maneira igualitária e buscando o bem comum, está ligada à ideia da formação da opinião pública e de sua importância enquanto mecanismo da sociedade civil para atuar como uma espécie de censora moralmente legitimada para tal tarefa. Observase com isso a interdependência da noção de democracia moderna com relação aos conceitos de esfera pública e de opinião pública.

A exposição acima, apesar de esquemática, nos fornece um pano de fundo sobre o qual podemos compreender os problemas trazidos por Fraser (1990). Como sugerido anteriormente, a metodologia trabalhada pela autora difere daquela utilizada por Habermas (2014). Enquanto este teoriza a esfera pública em seu aspecto histórico e normativo, Fraser (1990) estava preocupada com a sustentação do conceito em "actually existing democracy", isto é, nas contradições e impasses inerentes à democracia existente nas sociedades modernas. Com isso, Fraser (2014, p. 11) objetiva "repoliticize public sphere theory, which is currently [no contexto da globalização pós-Guerra Fria] in danger of being depoliticized"'3. É, inclusive, contra a despolitização da teoria que Fraser se ampara numa análise que combina teoria sociopolítica aos estudos culturais, uma vez que isso possibilitaria

[...] to study the ways in which culture is embedded in social structure and affected by social relations of domination. It thus provides an alternative to the sort of freefloating, decontextualized discourse analysis that dissociates cultural studies from critical social theory. (FRASER, 2002, p. 288) ${ }^{4}$

Assim, por ter em vista uma perspectiva crítico-teórica, Fraser não abandona totalmente o conceito de esfera pública como teorizado por Habermas (2014), especialmente no que concerne à distinção enfatizada por ele entre "state apparatuses, economic markets, and democratic associations, distinctions that are essential to democratic theory" ${ }^{\prime 5}$ (FRASER, 1990, p. 52). No entanto, a autora enxerga quatro pressupostos problemáticos que estariam subjacentes à concepção habermasiana de esfera pública.

\footnotetext{
3 "re-politizar a teoria da esfera pública, a qual está atualmente [no contexto da globalização pós-Guerra Fria] correndo risco de ser despolitizada." (FRASER, 2014, p. 11, tradução minha).

4 "[...] estudar as maneiras pelas quais a cultura é imbricada na estrutura social e afetada por relações sociais de dominação. Isso fornece uma alternativa ao discurso analítico descontextualizado e meio flutuante que dissocia estudos culturais de teoria social crítica." (FRASER, 2002, p. 288, tradução minha).

5 "aparatos estatais, mercados econômicos e associações democráticas, distinções que são essenciais para a teoria da democracia". (FRASER, 1990, p. 52, tradução minha).
} 
O primeiro pressuposto diz respeito ao acesso universal dos cidadãos à esfera pública. Nesse sentido, Fraser (1990) questiona a possibilidade de se colocar em suspenso as diferenças de status a fim de que haja deliberação em pé de igualdade entre interlocutores diferentes.

O segundo problema se refere à singularidade da esfera pública; ou seja, é possível pensar que haja uma única esfera pública? Ou estaríamos apenas enxergando a esfera pública oficial (ou hegemônica, nos termos de Warner [2002]) em detrimento de outras, com relações específicas com o Estado e com a sociedade civil?

O terceiro ponto criticado por Fraser (1990) está na pré-concepção verificada por ela na teoria de Habermas (2014) sobre o que seria o bem comum discutido na esfera pública e sua ideal separação dos interesses pessoais. Em outras palavras, a distinção entre bem comum e interesse pessoal não seria uma naturalização das concepções culturais do que é público e do que é privado?

Por fim, Fraser (1990) critica também o liberalismo que estaria latente na teoria de Habermas (2014) quando ele defende que a esfera pública democrática ideal requereria a separação nítida entre a sociedade civil e o Estado. A seguir, discutirei a primeira e a terceira questões de Fraser à teoria de Habermas, para depois refletir sobre o conceito da autora de contra-públicos subalternos, e sobre o liberalismo enxergado por ela na esfera pública habermasiana.

Situar a revisão de Fraser a Habermas significa, essencialmente, pensar a Modernidade (e seus processos formadores, como a esfera pública) segundo uma posição pós-moderna. É importante destacar a filiação de Fraser a um tipo de crítica desconstrucionista, pois a autora está comprometida com a desnaturalização dos discursos. Partindo de uma releitura da historiografia do humanismo iluminista sob essa perspectiva, constatou-se, a partir da segunda metade do século $\mathrm{XX}$, que os valores de universalidade e racionalidade do sujeito foram elaborados, no discurso da modernidade, baseados em uma autoimagem do homem ocidental construída por oposição a um outro de gênero sexual ou cor diferentes. Nas palavras de Meyda Yeğenoğlu (1998, p. 6): "In the Hegelian text, the subject represents itself to itself through the other and constitutes itself as universal, abstract subject (the I or ego) by signifying the other as a categorical opposite, a radical denial or negation of itself"6 ${ }^{\prime 6}$ O raciocínio de Fraser pode ser, portanto, associado a feministas pós-modernistas e a vários pesquisadores - entre eles Spivak (1999) e Mbembe (2014) - os quais se debruçaram sobre a noção hegeliana de sujeito para

\footnotetext{
6 "No texto hegeliano, o sujeito representa a si para si mesmo através do outro, e constitui-se como sujeito universal, abstrato (o eu ou o ego) pela significação do outro como uma categoria de oposição, uma negação ou rejeição radical de si mesmo." (YEĞENOĞLU, 1998, p. 6, tradução minha).
} 
estudar sua relação discursiva com a institucionalização da dominação colonial e do patriarcado.

Inserida nesse contexto crítico, Fraser censura com justeza o lugar coadjuvante da categoria de gênero sexual no livro de Habermas, e vai mais além ao posicionar o gênero não como um elemento adicional a ser analisado, mas enquanto fator crucial para a constituição da esfera pública liberal. Parafraseando Joan Landes, Fraser (1990, p. 59) traz o seguinte argumento:

For Landes, the key axis of exclusion is gender; she argues that the ethos of the new republican public sphere in France was constructed in deliberate opposition to that of a more woman-friendly salon culture that the republicans stigmatized as "artificial," "effeminate," and "aristocratic." Consequently, a new, austere style of public speech and behavior was promoted, a style deemed "rational," "virtuous," and "manly."7

Isso posto, voltemos ao primeiro e ao terceiro pontos, destacados na página anterior, sobre os questionamentos de Fraser (1990) a respeito da esfera pública habermasiana, quais sejam: sua acessibilidade universal e a rígida distinção entre esfera pública e privada.

Fraser inicia sua reflexão sobre a universalidade da esfera pública problematizando não apenas sua eficácia prática, como o faz Habermas ao refletir que esse ideal nunca teria sido alcançado de fato, mas principalmente seus pressupostos latentes. Assim, a utopia de que todos tenham acesso à esfera pública e que possam discutir o bem comum apesar de suas diferenças de status é, para Fraser, uma idealização liberalista, pois não ambiciona o confronto e a anulação das desigualdades, mas tão somente sua suspensão.

O problema apontado por Fraser (1990) está em que, mesmo que tomemos como base o acesso formal das minorias às arenas de deliberação, as desigualdades sociais e econômicas dos grupos subalternos são barreiras informalmente estruturadas no discurso e nas práticas socioculturais. Isto é, mesmo que em uma assembleia não haja nenhum impedimento legal ou formal para a participação igualitária de todos os seus participantes, pesquisas recentes demonstram que existem maneiras tão sutis de dominação que são facilmente mascaradas pela voz da maioria. Para ilustrar, a autora cita a pesquisa de Jane Mansbrigde (apud FRASER,

\footnotetext{
7 "Para Landers, o eixo central da exclusão é o gênero; ela argumenta que o ethos da esfera pública da nova república na França foi constituído em oposição deliberada àquela cultura de salão mais tolerante às mulheres que os republicanos estigmatizaram como sendo 'artificial', 'afeminada' e 'aristocrática'. Consequentemente, um novo e austero estilo de discurso e comportamento públicos foi promovido, um estilo considerado 'racional', 'eficiente' e 'masculino'". (FRASER, 1990, p. 59, tradução minha).
} 
1990), que verificou uma desigualdade de tempo e de valor nas falas de mulheres em comparação à fala masculina - mesmo em ambientes formalmente igualitários ${ }^{8}, \operatorname{logo}$

\begin{abstract}
Mansbridge rightly notes that many of these feminist insights into ways in which deliberation can serve as a mask for domination extend beyond gender to other kinds of unequal relations, like those based on class or ethnicity. They alert us to the ways in which social inequalities can infect deliberation, even in the absence of any formal exclusion. (FRASER, 1990, p. 64; grifo meu) ${ }^{9}$.
\end{abstract}

Se considerarmos como certo que tanto Habermas (2014) quanto Fraser (1990) têm em mente uma construção democrática e, portanto, coletiva da sociedade, para Habermas (2014) os indivíduos poderiam suspender discursivamente suas desigualdades a fim de deliberar sobre maneiras de, na prática, eliminá-las. Fraser (1990), no entanto, acredita que, dada a impossibilidade de suspender as desigualdades, seria mais eficaz que as deliberações fossem feitas em primeiro lugar a partir do confronto das desigualdades, evidenciando-as e buscando, antes de mais nada, desconstruí-las em um sentido socioeconômico para, só então, discutir o que seria de fato o bem comum (dentro de uma perspectiva não-liberalista) ${ }^{10}$.

Vimos que parte do problema da deliberação está relacionada com as desigualdades de status. Outra parte estaria, segundo Fraser, na oposição entre bem comum e interesses privados, subentendida na separação entre esfera pública e privada. Segundo Habermas (2014, p. 122123), as esferas públicas e privadas foram se delineando concomitantemente, por oposição mútua. Pensando a partir da sociedade contemporânea, na qual escreve o autor, é compreensível que ele idealizasse essa separação, uma vez que os interesses privados (enquanto interesses capitalistas/de mercado) não deveriam se misturar aos interesses públicos, no sentido da coletividade como um todo, como medida para evitar que grupos economicamente vulneráveis fossem prejudicados. Voltaremos a isso na conclusão, quando tratarei do liberalismo criticado por Fraser no livro de Habermas.

\footnotetext{
${ }^{8}$ Fraser (1990, p. 64) destaca, especificamente, as interrupções sistemáticas [manterrupting, em inglês] e a falta de interesse pelas falas femininas, bem como o problema de grupos minoritários para encontrarem uma linguagem pela qual se fazerem ouvidos.

9 "Mansbridge acertadamente observa que muitas dessas visões feministas sobre as formas pelas quais a deliberação pode servir como uma máscara para a dominação estendem-se além do gênero para outros tipos de relações desiguais, como aquelas baseadas em classe e etnia. Elas nos alertam para as maneiras pelas quais iniquidades sociais podem modular deliberações, mesmo na falta de qualquer exclusão formal." (FRASER, 1990, p. 64, grifo meu, tradução minha).

${ }^{10}$ Fraser (1990, p. 64) reconhece que Habermas, em trabalho posterior, concorda que seria melhor evidenciar as diferenças.
} 
Por enquanto, basta que tomemos a questão sobre outra ótica, do sentido de privado como aquilo que pertence à vida doméstica/individual em contraste com a vida pública. Nesse sentido é que Fraser problematiza a distinção entre as esferas, por reconhecer nela um discurso que naturaliza o que seria de interesse público. Coincidente com essa separação das esferas está a distinção de gênero. Segundo Jean Franco (1992), historicamente atribuíram-se, por um lado, a racionalidade, a escrita objetiva e a vida pública ao gênero masculino; por outro lado, o gênero feminino foi associado à sensibilidade, à oralidade, à escrita confessional e à privacidade do lar. O esforço de Fraser (1990), assim com o de Franco (1992), está, portanto, em desestabilizar a rigidez dessa distinção.

Afinal, como precisar a priori o que é de interesse comum? O sexo deveria mesmo estar totalmente restrito à esfera privada? A pergunta é relevante, uma vez que enquanto a homossexualidade permaneceu assunto individual foi difícil buscar uma linguagem que expressasse publicamente as reivindicações por direitos civis igualitários e por mudança sociocultural. Nesse exemplo, e no caso da violência doméstica (FRASER, 1990), notamos a necessidade de grupos minoritários exporem na esfera pública assuntos tidos como privados (por acontecerem dentro do espaço doméstico) e de, além disso, motivarem debates que, de início, não ganhavam interlocutores variados.

Chegamos finalmente à crítica de Fraser (1990) à noção habermasiana de esfera pública como algo singular (e universal). Segundo a autora, seria não somente factual, mas também preferível, que houvesse várias esferas públicas ao invés de uma só. Sobre o aspecto empírico do que ela chama de contra-públicos subalternos, podemos resgatar a leitura pós-moderna da Modernidade para argumentar que esse processo, ligado à ascensão da burguesia capitalista, foi fundamentalmente excludente para todos aqueles que não se conformassem à imagem do sujeito universal como motor civilizatório do mundo. A esfera pública oficial, como a denomina Fraser (1990), teria traçado um processo semelhante ao da Modernidade, como sugerido acima, ao se estabelecer como dominante em relação de exclusão a outros tipos de esferas públicas, tornadas subalternas:

Virtually from the beginning, counterpublics contested the exclusionary norms of the bourgeois public, elaborating alternative styles of political behavior and alternative norms of public speech. Bourgeois publics, in turn, excoriated these alternatives and deliberately sought to block broader participation. As [Geoff] Eley puts it, "the emergence of a bourgeois public was never defined solely by the struggle against absolutism and traditional authority, but... addressed the problem of popular 
containment as well. The public sphere was always constituted by conflict." (FRASER, 1990, p. 61) ${ }^{11}$.

Desse modo, os contra-públicos - esferas públicas alternativas - teriam sido constituídos de maneira ao mesmo tempo paralela e conflitante com a esfera pública oficial. De acordo com Achille Mbembe:

Situados à força num mundo à parte, reservando as suas qualidades de seres humanos para lá da submissão, aqueles que tinham sido adornados com o nome de "negro" [...] foram obrigados a fundar suas próprias instituições - escolas, jornais, organizações políticas, uma esfera pública que nada tem a ver com a esfera pública oficial. (MBEMBE, 2014, p. 91-92)

Michael Warner (2002), em seu artigo sobre a relação discursiva entre a esfera pública hegemônica e seus contra-públicos, reconhece a contribuição de Fraser à crítica sobre a singularidade e a universalidade da esfera pública, mas diverge da autora em sua caracterização desses contra-públicos como essencialmente subalternos. De fato, conforme argumenta Warner (2002), não há nada de propriamente subalterno no cristianismo fundamentalista estadunidense ou na cultura jovem, por exemplo, mesmo que tais grupos possam ser compreendidos como subordinados à esfera pública hegemônica.

Trata-se de uma crítica importante, pois a ideia de subalternidade, como conceituada por Spivak (2010) e diversos intelectuais latino-americanos (ACHUGAR; BEVERLY, 1992), está ligada a uma combinação entre a impossibilidade de produção e/ou circulação independente da própria fala e a categorias de gênero, classe e cor historicamente marginalizadas. Por isso, mesmo que os discursos produzidos por grupos neonazistas, por exemplo, não possam ser caracterizados como hegemônicos, eles, por outro lado, também não podem ser tidos por subalternos, mas apenas como contra-públicos ou simplesmente subpúblicos (WARNER, 2002).

Por fim, gostaria de me concentrar à crítica de Fraser (1990, p. 74-77) acerca do que ela chama de concepção burguesa da esfera pública, a qual idealiza a separação entre Estado e sociedade civil como condição para o bom funcionamento das democracias. Segundo a autora, precisamos primeiramente considerar a "sociedade civil" segundo duas acepções, a) enquanto

\footnotetext{
11 "Praticamente desde o início, contra-públicos contestaram as normas excludentes do público burguês, elaborando estilos alternativos de comportamento político e normas alternativas de discurso público. Os públicos burgueses, por sua vez, censuraram essas alterativas e deliberadamente procuraram bloquear uma participação mais ampla. Como [Geoff] Eley coloca, 'a emergência de um público burguês nunca foi definida exclusivamente pela luta contra o absolutismo e a autoridade tradicional, mas... também pela abordagem de problemas de contenção popular. A esfera pública sempre foi constituída por conflito."' (FRASER, 1990, p. 61, tradução minha).
} 
sociedade de mercado e b) enquanto associação ao mesmo tempo não-governamental e "nãomercantil" de indivíduos.

No primeiro caso, essa separação entre Estado e sociedade corresponderia ao liberalismo clássico, segundo o qual o Estado não precisaria, nem deveria, intervir nos assuntos ou negócios proveniente da iniciativa privada. Assim, a sociedade civil alcançaria seu desenvolvimento de maneira independente, com lógicas e regras próprias, garantindo certo equilíbrio socioeconômico. O segundo caso, por outro lado, corresponderia ao ideal de uma democracia em que os indivíduos, através de suas livres deliberações na esfera pública, formam uma opinião pública crítica das ações do Estado, o qual, por sua vez, teria que levar a sério as avaliações e demandas provenientes da sociedade, sob risco de perder sua legitimidade.

Sobre essa segunda definição, e ainda considerando a noção dos contra-públicos como espaços nos quais os interesses de um dado grupo social podem ser melhor articulados, Fraser questiona, entre outras coisas, se o impacto dessas deliberações civis não estaria prejudicado pela falta de mecanismos mais adequados para a participação ativa dos indivíduos nas ações do Estado, tendo em vista que:

[...] any conception of the public sphere that requires a sharp separation between (associational) civil society and the state will be unable to imagine the forms of selfmanagement, inter-public coordination, and political accountability that are essential to a democratic and egalitarian society. The bourgeois conception of the public sphere, therefore, is not adequate for contemporary critical theory. What is needed, rather, is a post-bourgeois conception that can permit us to envision a greater role for (at least some) public spheres than mere autonomous opinion formation removed from authoritative decision-making. (FRASER, 1990, p. 76) ${ }^{12}$

Com isso, mais do que precisar os contornos de uma (nova) forma política inclusiva, na qual as esferas públicas tivessem mais força de atuação, Fraser ambiciona tornar visíveis os limites que essa separação impõe às democracias existentes, bem como problematizar a naturalidade com que se toma a separação entre Estado e sociedade na teoria habermasiana, e a correspondência dessa abordagem com a filosofia liberalista.

Nesse sentido, concorda-se com Nancy Fraser de que o conceito de esfera pública habermasiano, apesar de poder se configurar como um horizonte de expectativa do

12 "[...] qualquer concepção de esfera pública que requeira uma separação rígida entre sociedade civil (associativa) e o Estado será incapaz de imaginar as formas de autogerenciamento, coordenação inter-pública e responsabilidade política que são essenciais para uma sociedade democrática e igualitária. A concepção burguesa de esfera pública, portanto, não é adequada para a teoria crítica contemporânea. O que é preciso, ao invés disso, é uma concepção pós-burguesa que nos permita vislumbrar para (ao menos) as esferas públicas um papel maior que o da mera formação de opinião autônoma afastada de tomadas de decisões autoritárias." (FRASER, 1990, p. 76, tradução minha). 
desenvolvimento democrático das sociedades liberais, é problemático porque pouco inclusivo. Assim sendo, as contribuições de Fraser, feitas a partir de uma perspectiva feminista preocupada com a participação ativa de mulheres e demais grupos minoritários na construção de uma democracia pós-liberal, são essenciais para se ampliar o debate sobre o acesso igualitário aos espaços de formulação de questões e de decisão.

\section{REFERÊNCIAS BIBLIOGRÁFICAS}

ACHUGAR, Hugo; BEVERLY, John (Ed.). La voz del otro: testimonio, subalternidad y verdad narrativa. Lima, Pittsburgh: Latinoamericana Editores, 1992.

DUMONT, Louis. $O$ individualismo: uma perspectiva antropológica da ideologia moderna. Tradução: Álvaro Cabral. Rio de Janeiro: Rocco, 1985.

FRANCO, Jean. Si me periten hablar: La lucha por el poder interpretativo. In: ACHUGAR, Hugo; BEVERLY, John (Ed.). La voz del otro: testimonio, subalternidad y verdad narrativa. Lima, Pittsburgh: Latinoamericana Editores, 1992.

FRASER, Nancy. Rethinking the Public Sphere: A Contribution to the Critique of Actually Existing Democracy. Social Text, n. 25/26, p. 56-80, 1990.

Politics, culture, and the public sphere: toward a postmodern conception. In: NICHOLSON, Linda; STEVEN, Seidman. Social postmodernism: beyond identity politics. Cambrigde: Cambrigde University Press, 2002. p. 287-312.

HABERMAS, Jürgen. Mudança Estrutural da Esfera Pública: investigações sobre uma categoria da sociedade burguesa. Trad. Denilson Luís Werle. 1 ed. São Paulo: Editora Unesp, 2014.

MBEMBE, Achille. Crítica da razão negra. Trad. Marta Lança. 1 ed. Lisboa: Antígona, 2014.

SPIVAK, Gayatri Chakravorty. A critique of postcolonial reason: toward a history of the vanishing present. Londres: Harvard University Press: 1999.

. Pode o subalterno falar? Trad. Sandra R. G. Almeida, Marcos P. Feitosa, André P. Feitosa. Belo Horizonte: Editora UFMG, 2010.

WARNER, Michael. Publics and counterpublics. Public Culture, v. 14, n. 1, p. 49-90, Winter, 2002.

YEĞENOĞLU, Meyda. Colonial Fantasies: towards a feminist reading of Orientalism.

Cambridge: Cambridge University Press, 1998. 